\title{
MAGDAS for Geospace Environment Monitoring
}

\author{
By Kiyohumi YumOTO ${ }^{1)}$, Hideaki KAWANO ${ }^{1)}$ and MAGDAS Group \\ ${ }^{1)}$ Space Environment Research Center, Kyushu University, Fukuoka, 812-8581, Japan
}

(Received April 25th, 2008)

\begin{abstract}
MAGnetic Data Acquisition System (MAGDAS) of the Circum-pan Pacific Magnetometer Network (CPMN) is introduced. MAGDAS/CPMN measures the ground magnetic field all around the world and sends the measured data to Space Environment Research Center (SERC), Fukuoka, Japan, via Internet, telephone line or satellite phone line. As examples of the phenomena observed by MAGDAS/CPMN, we present observations of the equatorial electrojet, Pi 2 waves near the dip equator, and SC-associated ionospheric electric field; Pi 2 is a transient ULF wave taking place at the beginning of a phenomenon called substorm, and SC (sudden commencement) is a phenomenon taking place as an interplanetary shock arrives at the Earth. We also compare ground-based estimations of the space plasma density with simultaneous direct observations by spacecraft.
\end{abstract}

Key Words: MAGDAS/CPMN, Ground-based Observations, Equatorial Electrojet, Pi 2, SC

\section{Introduction}

The Space Environment Research Center (SERC), Kyushu University is deploying the MAGnetic Data Acquisition System (MAGDAS) at the Circum-pan Pacific Magnetometer Network $(\mathrm{CPMN})^{1)}$ consisting of 50 stations, and several FM-CW radars ${ }^{2)}$ along the $210^{\circ}$ magnetic meridian, in collaborations with many organizations in the world. The MAGDAS project has the potential to contribute greatly to IHY (International Heliophysical Year) by supporting ground-based magnetometer array for worldwide studies for space weather ${ }^{3,4)}$, and by demonstrating the beauty, importance, and relevance of space science to the world.

The goal of MAGDAS is to become the most comprehensive ground-based monitoring system of the Earth's magnetic field. It does not compete with space-based observation. Rather, this ground-based

\section{MAGDAS/CPMN}

(MAGnetic Data Acqusition System/Circum-pan Pacific Magnetometer Network)

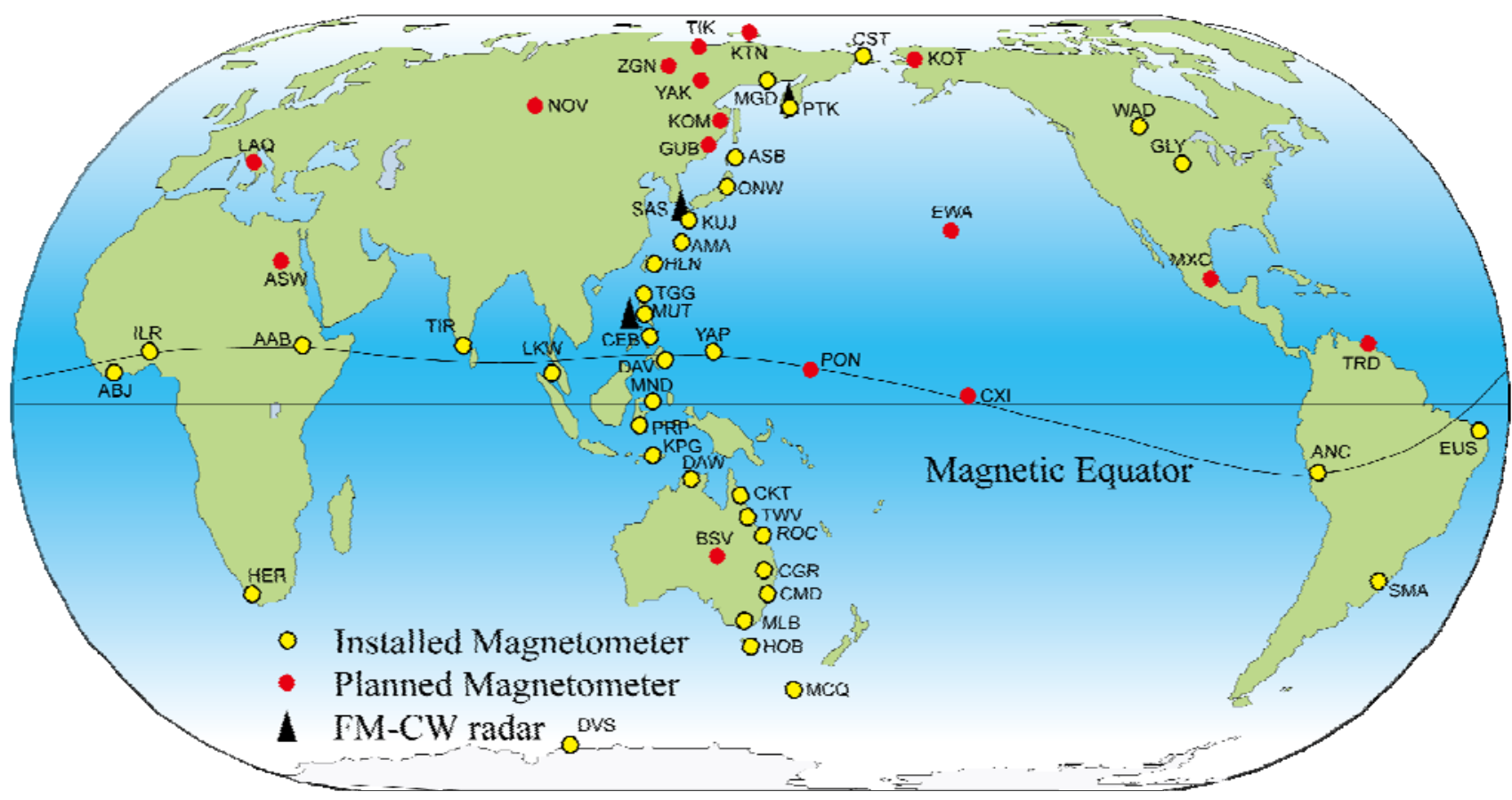

Fig. 1. Map of MAGDAS/CPMN 
network complements observation from space. To properly study solar-terrestrial events, data from both are required.

This paper summarizes the MAGDAS/CPMN system and presents a few results obtained from the MAGDAS project.

\section{The MAGDAS/CPMN system}

The MAGDAS/CPMN system consists of two main parts. The MAGDAS-A system is a new magnetometer system installed at the CPMN stations, while MAGDAS-B is the data acquisition and monitoring system installed at SERC. The new magnetometer system (MAGDAS-A) consists of a sensor unit, data logging/transferring units, and a power unit. The sensor unit includes three-axial fluxgate-type ring-core sensors, tilt meters, and a thermometer. The total weight of MAGDAS-A is less than $15 \mathrm{~kg}$. The data transferring unit transfers 1 -sec averaged data $(H+\delta H, D+\delta D, Z+\delta Z, F+\delta F$, where $H, D$, and $Z$ are three components of the magnetic field vector, and $F$ is the magnetic field strength) in real time from overseas stations to SERC, Japan, by using three possible ways: The Internet, the telephone line or the satellite phone line.

Nearly 20 MAGDAS units were installed along the $210^{\circ}$ magnetic meridian in 2005 . In and after 2006, nearly 20 MAGDAS units were installed along the magnetic equator, and at places around the Earth such as North America, South Africa, Siberia, Antarctica, and Egypt. The MAGDAS project is ongoing, and MAGDAS units will be deployed around the Earth in places such as Mexico, Italy, Alaska, and Africa.

\section{New findings with the MAGDAS/CPMN data}

By analyzing magnetic field data from these MAGDAS units, we can real-time monitor and model three-dimensional global distributions of the electric current and the plasma mass density in the geospace. The analysis results help us understand changes in the electromagnetic and plasma environment of the geospace during helio-magnetospheric storms. In the following, we will present a few results of the MAGDAS data analyses.

\subsection{Equatorial electrojet}

In order to understand couplings of the solar wind-magnetosphere-ionosphere-atmosphere system, data from an equatorial MAGDAS station DAV (Dip Latitude $=-0.65^{\circ}$, Magnetic Longitude $=196.54^{\circ}$, solar wind parameters, and geomagnetic indices, for the interval from Jul. 1, 2005 to Mar. 4, 2006, were spectral analyzed $^{5)}$. As a result, the Fourier Transform (FT) of $(H(\mathrm{DAV})-D s t)$, where $H(\mathrm{DAV})$ refers to the magnetic field $H$ component at DAV and Dst refers to the Dst index, had peaks at the periods of 7.5 day and 14.5 day; these peaks are not included in any FT's of the geomagnetic indices and solar wind parameters. These spectrum peaks are suggested to come from strong neutral wind-plasma interactions in the mesosphere-ionosphere.

\subsection{Pi 2 wave characteristics near the dip equator}

Pi 2 is a transient ULF wave observed all around the world at the beginning of a phenomenon called substorm ${ }^{6)}$. By using the equatorial Pi 2 pulsations observed at the world-widely distributed stations near the dip equator at ILR (Dip Lat. $=-2.95^{\circ}$, M. Long $=76.80^{\circ}$ ), $\operatorname{AAB}\left(0.56^{\circ}, 110.47^{\circ}\right), \operatorname{CEB}\left(2.73^{\circ}, 195.06^{\circ}\right), \operatorname{ANC}\left(0.72^{\circ}\right.$, $\left.354.33^{\circ}\right)$, and $\operatorname{EUS}\left(-7.00^{\circ}, 34.21^{\circ}\right)$, we have found the following wave characteristics: Pi 2 pulsations observed near the dip equator show an amplitude enhancement around 10:00-13:00 local time. If Site $\mathrm{A}$ is closer to the dip equator than Site B, Site A observes Pi 2 amplitudes larger than those at Site B. The Pi 2 amplitude tends to increase with decreasing ambient field intensity.

The above-stated enhancement near the noon meridian takes place because the ionospheric conductivity is the largest near the noon meridian. That is, because the sunlight ionizes the neutral particles in the ionosphere, and because the sunlight is almost vertically incident on the ionosphere near the noon meridian, the ionization effect of the sunlight is the largest near the noon meridian. It means that the ionospheric conductivity is the largest near the noon meridian, which means that, for an electric field of Pi 2 incident from space to the ionosphere, the corresponding ionospheric current is the largest near the noon meridian. The ground magnetic field is enhanced near the noon meridian due to this enhanced ionospheric current.

\subsection{SC-associated ionospheric electric field}

It is well known that, when an interplanetary shock arrives at the Earth from the Sun, a phenomenon called sudden commencement (SC), related to the compression of the magnetosphere, takes place ${ }^{7)}$. When an SC takes place, MAGDAS observes a step-like increase in the magnetic field strength, and the FM-CW radar in Sasaguri, Fukuoka, Japan (M. Lat. $=23.2^{\circ}, \quad$ M. Long=199.6 ${ }^{\circ}$ ) observes a step-like change in the ionospheric electric field, at the same time. From the analysis of the SC-associated ionospheric electric field observed by the FM-CW radar in Sasaguri, we have found that the westward component of the ionospheric electric field is stronger in nighttime than in daytime. This result can be explained if the magnetosphere-wide dawn-to-dusk SC electric field is superimposed on the westward electric field due to the earthward compression of the ionosphere; the two types of the electric field are both caused by the interplanetary shock at the same time ${ }^{2)}$. If we go into the details of the second of the above-stated two effects, the earthward compression of the ionosphere leads to the earthward (i.e., vertically downward) motion of the ionospheric plasma, which motion causes an ionospheric electric field which satisfies the frozen-in law; because the geomagnetic field is directed northward, the electric field $\mathbf{E}$, calculated by $\mathbf{E}=-\mathbf{v} \times \mathbf{B}$, is directed westward.

3.4. Conjunction observations of the magnetospheric plasma density both from the ground and in space

Data from CPMN ground magnetometers at TIK (M. Lat. $=65.8^{\circ}$, M. Long $=197.2^{\circ}$ ) and CHK (M. Lat. $=64.8^{\circ}$, M. Long $=212.5^{\circ}$ ) were analyzed by using the methods called "amplitude-ratio method" and 
"cross-phase method" to identify eigenoscillations of the geomagnetic field line running through the midpoint of the two stations ${ }^{8)}$. Here the term "eigenoscillation," or in orther words "normal mode oscillation," refers to the oscillation of a system in which all parts of the system oscillate with the same frequency. From the frequencies of thus identified eigenoscillations, the plasma mass density along the above-stated field line was estimated by using a field-line tracing method ${ }^{9)}$. Then, among thus identified eigenoscillation events, events were further selected so that, for the selected events, the Cluster spacecraft was located on the same field line at the event time. For thus selected ground-space conjunction events, the above-obtained plasma mass density was compared with the electron density measured by Cluster ${ }^{10)}$. This was the first such direct ground-space comparison.

As a result of the above-stated comparison, it was found that the two quantities were proportional in the first-order approximation, as expected. Further it was found that the ratio of the mass density to the electron number density was larger when the geomagnetic activity was larger (such as in magnetic storms); this is consistent with past satellite observations showing that the relative abundance of heavy ions (such as $\mathrm{O}+$ ) was large during geomagnetically active times ${ }^{11)}$

It often happens that, due to a satellite-mass limitation, a satellite does not carry an instrument which can measure the ion composition while it carries an instrument which measures the electron number density. For such cases, ground-based simultaneous measurements can provide information on the ion composition in space in the above-stated manner.

It is also to be noted that, while it is difficult to have many scientific satellites measuring the magnetosphere at the same time because satellites are expensive, we already have 50 ground magnetometers in the world running at the same time; thus we can simultaneously monitor wider area of space from the ground, and the obtained information can fill the gap that are not directly monitored by satellites. This is the advantage of ground-based observations, and at the same time, ground-satellite collaborations are useful because satellites provide direct and detailed information from in situ measurements of space.

\section{Summary}

In this paper we have introduced MAGDAS/CPMN (MAGnetic Data Acquisition System at Circum-pan Pacific Magnetometer Network). Data from the MAGDAS/CPMN magnetometers, placed all around the world, are being sent to SERC (Space Environment Research Center), Kyushu University, Japan in a real-time manner by using Internet lines, telephone lines, or satellite phone lines.

We have made new findings by using thus obtained data. This paper introduces in Sections 3.1 through 3.3 newly found ionospheric features of the equatorial electrojet, Pi 2, and a sudden commencement; because the ionosphere and the magnetosphere are connected via geomagnetic field lines, the phenomena taking place in the ionosphere are related to phenomena in the magnetosphere. In this paper we have mentioned expected magnetospheric features corresponding to the newly found ionospheric features, and the actual study of corresponding spacecraft data in the magnetosphere is a topic of future research.

Section 3.4 of this paper introduces a study of ground-space comparisons by using the MAGDA/CPMN data and data from Cluster spacecraft; ground-based estimates of the magnetospheric plasma density are shown to be reliable. The wide coverage of the Earth surface by ground magnetometers will enable us to monitor the global distribution of the magnetospheric plasma density, while satellites (that are much smaller in number than ground magnetometers) can provide detailed information at the satellite positions; combination of the two types of observations is thus useful.

\section{References}

1) Yumoto, K., and the $210^{\circ} \mathrm{MM}$ Magnetic Observation Group: The STEP $210^{\circ}$ magnetic meridian network project, J. Geomag. Geoelectr., 48 (1996), pp.1297-1309.

2) Ikeda, A., K. Yumoto, M. Shinohara, K. Nozaki, A. Yoshikawa and A. Shinbori: SC-associated ionospheric electric fields at low latitude: FM-CW radar observation, Memoirs of the Faculty of Sciences, Kyushu University, Ser. D. Earth and Planet. Sci., XXXII (2008), pp.1-6.

3) Yumoto, K. and the MAGDAS Group: MAGDAS project and its application for space weather, Solar Influence on the Heliosphere and Earth's Environment: Recent Progress and Prospects, Edited by N. Gopalswamy and A. Bhattacharyya, ISBN-81-87099-40-2 (2006), pp. 309-405.

4) Yumoto K. and the MAGDAS Group: Space weather activities at SERC for IHY: MAGDAS, Bull. Astr. Soc. India, 35 (2007), pp. 511-522.

5) Uozumi, T., K. Yumoto, K. Kitamura, S. Abe, Y. Kakinami, M. Shinohara, A. Yoshikawa, H. Kawano, T. Ueno, T. Tokunaga, D. McNamara, J. K. Ishituka, S. L. G. Dutra, B. Damtie, V. Doumbia, O. Obrou, A. B. Rabiu, I. A. Adimula, M. Othman, M. Fairos, R. E. S. Otadoy, and MAGDAS Group: A new index to monitor temporal and long-term variations of the Equatorial Electrojet by MAGDAS/CPMN real-time data: EE-Index, Earth Planets Space, 60 (2008), pp.785-790.

6) Yumoto, K., and the CPMN Group: Characteristics of Pi 2 magnetic pulsations observed at the CPMN stations: A review of the STEP results, Earth Planets Space, 53 (2001), pp.981-992.

7) Araki, T.: Global structure of geomagnetic sudden commencements, Planet. Space Sci., 25 (1977), pp.502-506.

8) Maeda, N., S. Takasaki, H. Kawano, S. Ohtani, P. M. E. Decreau, J. G. Trotignon, S. I. Solovyev, D. G. Baishev, and K. Yumoto, Simultaneous observations of the plasma density on the same field line by the CPMN ground magnetometers and the Cluster satellites, Advances in Space Research, in press, doi:10.1016/j.asr.2008.04.016.

9) Singer, H. J., D. J. Southwood, R. J. Walker, and M. G. Kivelson, Alfven wave resonances in a realistic magnetospheric magnetic field geometry, J. Geophys. Res., 86 (1981), pp.4589-4596.

10) Décréau, P. M. E., P. Fergeau, V. Krannosels'kikh, M. Lévêque, Ph. Martin, O. Randriamboarison, F. X. Sené, J. G. Trotignon, P. Canu, and P. B. Mögensen, Whisper, a resonance sounder and wave analyzer: performances and perspectives for 
the Cluster mission, Space Sci. Reviews, 79 (1997), pp.157-193.

11) Daglis L. A., G. Kasotakis, E. T. Sarris, Y. Kamide, S. Livi, and B. Wilken, Variations of the ion composition during an intense magnetic storm and their consequences, Phys. Chem. Earth, 24 (1999), pp.229-232. 